\title{
Bile acids initiate cholestatic liver injury by triggering a hepatocyte-specific inflammatory response
}

\author{
Shi-Ying Cai, ${ }^{1}$ Xinshou Ouyang, ${ }^{1}$ Yonglin Chen, ${ }^{1}$ Carol J. Soroka, ${ }^{1}$ Juxian Wang, ${ }^{2}$ Albert Mennone, ${ }^{1}$ \\ Yucheng Wang, ${ }^{2}$ Wajahat Z. Mehal, ${ }^{1}$ Dhanpat Jain, ${ }^{1}$ and James L. Boyer ${ }^{1}$ \\ 'Department of Internal Medicine and Yale Liver Center, Yale University School of Medicine, New Haven, Connecticut, USA. \\ ${ }^{2}$ Institute of Medicinal Biotechnology, Chinese Academy of Medical Sciences and Peking Union Medical College, \\ Beijing, China.
}

\begin{abstract}
Mechanisms of bile acid-induced (BA-induced) liver injury in cholestasis are controversial, limiting development of new therapies. We examined how BAs initiate liver injury using isolated liver cells from humans and mice and in-vivo mouse models. At pathophysiologic concentrations, BAs induced proinflammatory cytokine expression in mouse and human hepatocytes, but not in nonparenchymal cells or cholangiocytes. These hepatocyte-specific cytokines stimulated neutrophil chemotaxis. Inflammatory injury was mitigated in $\mathrm{Ccl}^{-1-}$ mice treated with BA or after bile duct ligation, where less hepatic infiltration of neutrophils was detected. Neutrophils in periportal areas of livers from cholestatic patients also correlated with elevations in their serum aminotransferases. This liver-specific inflammatory response required BA entry into hepatocytes via basolateral transporter Ntcp. Pathophysiologic levels of BAs induced markers of ER stress and mitochondrial damage in mouse hepatocytes. Chemokine induction by BAs was reduced in hepatocytes from $\mathrm{TIr}^{-1-}$ mice, while liver injury was diminished both in conventional and hepatocyte-specific $\mathrm{Tl}^{\mathrm{r} \mathrm{g}^{-/-}}$mice, confirming a role for Tlrg in BA-induced liver injury. These findings reveal potentially novel mechanisms whereby $B A s$ elicit a hepatocyte-specific cytokine-induced inflammatory liver injury that involves innate immunity and point to likely novel pathways for treating cholestatic liver disease.
\end{abstract}

Conflict of interest: The authors have declared that no conflict of interest exists.

Submitted: September 19, 2016 Accepted: January 19, 2017 Published: March 9, 2017

Reference information: JCI Insight. 2017;2(5):e90780. https:// doi.org/10.1172/jci.insight.90780.

\section{Introduction}

Bile formation is a vital function of the liver in all vertebrates. Bile acids (BAs) are the major constituents of bile. They stimulate bile secretion and are required for the intestinal absorption of lipids and fat-soluble vitamins. BAs also modulate gene expression through signal transduction pathways that regulate lipid metabolism and energy expenditure (1). However, when BA excretion is disrupted, they accumulate in the liver and systemic circulation and cause cholestatic liver injury that often leads to progressive liver damage and subsequent liver failure. Despite this well-known sequence of events, the molecular mechanisms by which BAs injure the liver remain unclear. One favored hypothesis is that toxic BAs induce hepatocyte apoptosis by activating death receptors (2). This assumption is based primarily on in vitro observations from cultured cells that were exposed to high concentrations of toxic BAs $(3,4)$. However, such high concentrations are rarely achieved in the serum of cholestatic patients and animal models $(5,6)$. Most importantly, apoptosis of hepatocytes has not been detected in vivo in the liver of bile duct-ligated (BDL) mice or in vitro in BA-treated human hepatocytes $(7,8)$, suggesting that BA-induced liver injury must occur by alternative mechanisms. Recent studies suggest that BA may induce liver injury via an inflammatory response, as neutrophil infiltration correlated with the injury in cholestatic rodent models $(9,10)$. This notion is further strengthened by the observation that BA stimulated the expression of inflammatory cytokines in cultured mouse hepatocytes $(6,11)$. This induction is partially dependent on the transcription factor early growth response 1 (Egr1), although it is not clear how BAs activate Egr1. Elevated levels of cytokines such as Ccl2, Cxcl2, and IL-8 were also detected in the serum and liver of patients with cholestasis and in cholestatic animal models $(6,11-13)$. Antagonizing inflammatory cytokines reduced liver injury in BDL mice (14-16), further supporting the notion of the role of the inflammatory response in 
cholestatic liver injury. However, the cellular source of BA-induced cytokines, particularly as liver injury from cholestasis is first initiated, remains unclear, as do the intracellular signaling pathways.

Members of the Toll-like receptor (TLR) family play a critical role in tissue-damage-initiated inflammatory responses to both endogenous sterile insults and pathogen recognition. TLR9 has been characterized as an intracellular DNA receptor that, upon activation, stimulates cytokine production as part of the innate immune response of the host to pathogens or damage-associated molecular patterns (17). Mitochondrial DNA (mtDNA) has been identified as a damage-associated molecular pattern that can activate Tlr9 (18). High levels of BA have been shown to injure mitochondria by changing their membrane potential and releasing cytochrome $c(19)$. Thus, mitochondrial damage and the release of mtDNA caused by BAs might lead to activation of TLR responses, but this possibility has not been examined.

In this report, we addressed these questions in vitro using isolated liver cells from mouse and human, and in vivo using cholestatic mouse models with WT and gene-knockout (KO) mice. We found that hepatocytes, but not nonparenchymal cells (NPCs), released inflammatory cytokines that stimulated neutrophil chemotaxis when treated with pathophysiological levels of endogenous BAs. This inflammatory response involves mitochondrial damage and Tlr9 activation in mouse hepatocytes. Inflammation and liver injury were significantly reduced in vivo in the absence of Tlr9 or Ccl2 in animal models of cholestasis. Periportal neutrophil infiltrates correlated with serum aminotransferase elevations in cholestatic patients, further supporting a role for inflammation in this liver injury in humans. These findings provide a potentially novel sequential mechanism for the initiation of BA-induced liver injury in cholestasis, and point to several potential therapeutic interventions.

\section{Results}

Hepatocytes, but not NPCS, stimulated mRNA expression of inflammatory cytokines in response to BA treatment. Hepatocytes, cholangiocytes, and liver NPCs were isolated from mice and cultured. As demonstrated in Figure 1A, $100 \mu \mathrm{M}$ taurocholic acid (TCA), the major endogenous BA in rodents, significantly increased mRNA expression of the inflammatory cytokines, $\mathrm{Ccl} 2$ and $\mathrm{Cxcl} 2$ in hepatocytes, but not in NPCs. In contrast, LPS greatly stimulated the expression of these genes in both hepatocytes and NPCs, whereas tauroursodeoxycholic acid (TUDCA), a therapeutic BA, did not affect the expression of these cytokines in either cell population. In addition, TCA did not induce $\mathrm{Ccl} 2$ and $\mathrm{Cxcl} 2$ expression in cultured mouse cholangiocytes or macrophages (Supplemental Figure 1; supplemental material available online with this article; https://doi.org/10.1172/jci. insight.90780DS1). TCA induction of $\mathrm{Ccl} 2$ and $\mathrm{Cxcl} 2 \mathrm{mRNA}$ expression was also time- and dose-dependent in mouse hepatocytes (Figure 1B). Furthermore, a spectrum of major endogenous BAs across vertebrate evolution also stimulated the expression of $\mathrm{Cxcl} 2$ in mouse hepatocytes, but the farnesoid X receptor (FXR) agonist GW4064 did not (Figure 1C). Even a 24-hour treatment with unconjugated BA, i.e., cholic acid (CA) and chenodeoxycholic acid (CDCA) (both up to $100 \mu \mathrm{M}$ ), failed to substantially stimulate Cxcl 2 mRNA expression in these cells (data not shown). Figure 1D shows that human hepatocytes have similar responses when treated with glycochenodeoxycholic acid (GCDCA), the major endogenous BA in humans. Twentyfour-hour treatment with $50 \mu \mathrm{M}$ GCDCA significantly increased CCL2 CCL15, CCL20, CXCL1, and IL-8 mRNA expression in these cells. Together, these findings indicate that pathophysiologic amounts of BA initiate an increase in expression of proinflammatory cytokines that is hepatocyte specific.

Inflammatory cytokines released from BA-treated hepatocytes stimulated neutrophil chemotaxis. To assess whether this BA-stimulated mRNA expression of inflammatory cytokines in hepatocytes was functionally significant, we first measured $\mathrm{Ccl} 2$ and $\mathrm{Cxcl} 2$ protein concentrations in the cell culture medium. As shown in Figure 2A, when hepatocytes were exposed to TCA or LPS, significantly higher amounts of Ccl2 and Cxcl2 protein were seen than in the medium control or after TUDCA treatment. In contrast, these proteins were not increased in the media from NPCs when treated with TCA or TUDCA, whereas elevated levels of these proteins were detected in LPS-treated cell culture medium (Figure 2A). Furthermore, when cell migration assays were performed, more neutrophils migrated across the transwell membranes when culture media were added from hepatocytes treated with TCA or LPS than when treated with TUDCA or the medium control (Figure 2B). Once again, there was no difference in neutrophil migration among culture media from NPCs treated with TCA, TUDCA, or the medium control, whereas more cells were seen in NPC medium treated with LPS (Figure $2 \mathrm{~B}$ ). Together, these results demonstrated that when hepatocytes were exposed to pathophysiologic levels of BA, they released cytokines as chemoattractants, which were capable of initiating neutrophil chemotaxis that then resulted in an inflammatory injury response. 
A

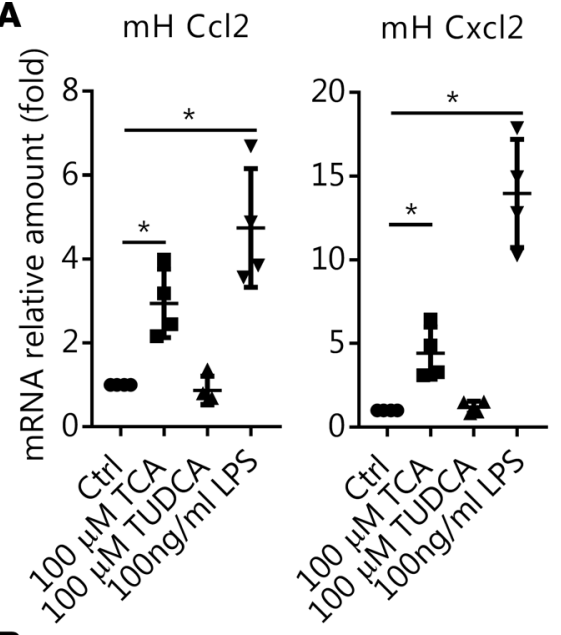

B
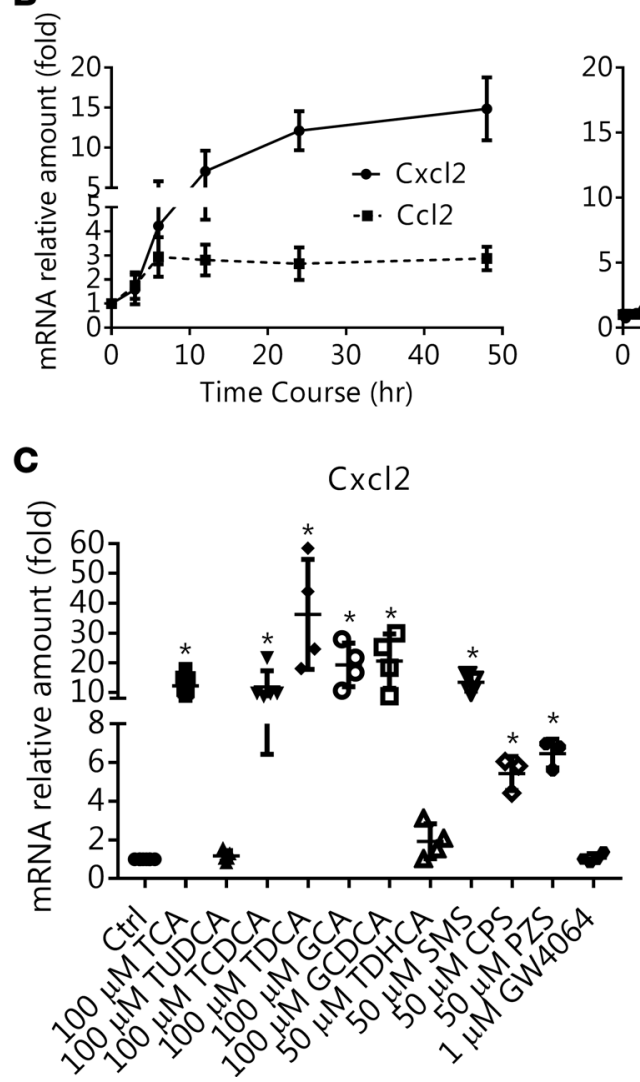

$\mathrm{mNPC} \mathrm{CCl2}$

$\mathrm{mNPC} \mathrm{Cxcl} 2$
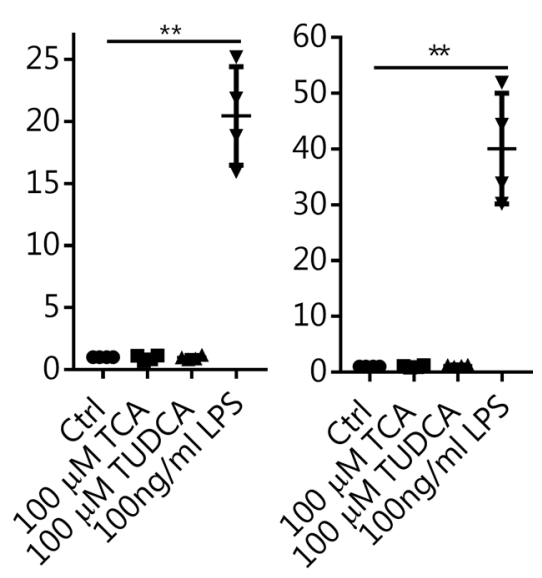

Figure 1. Bile acids stimulate chemokine mRNA expression in both human and mouse hepatocytes. (A) Mouse hepatocytes $(\mathrm{mH})$ and nonparenchymal cells (NPCs) were treated with $100 \mu \mathrm{M}$ taurocholic acid (TCA), tauroursodeoxycholic acid (TUDCA), or LPS for 6 hours. Results are the mean $\pm \mathrm{SD}, n \geq 4$. ${ }^{*} P$ $<0.05,{ }^{* *} P<0.01$ vs. medium control (Ctrl). (B) Bile acid-induced cytokine expression in mouse hepatocytes was time (100 $\mu \mathrm{M}$ TCA) and dose dependent. Hepatocytes were treated for 6 hours to demonstrate dose dependency of $\mathrm{Cc} 2 \mathrm{2}$ expression and 24 hours to show dose dependency for expression of $\mathrm{CxCl} 2$ (mean $\pm S D, n \geq 4$ ). (C) A number of different major vertebrate-endogenous bile salts also induced $\mathrm{CxCl} 2$ mRNA expression in mouse hepatocytes (24-hour treatment, mean $\pm S D$, ${ }^{*} P<0.05$ vs. Ctrl, $\left.n \geq 4\right)$. CPS cryprinol sulfate; GCA, glycocholic acid; GCDCA, glycochenodeoxycholic acid; PZS, petromyzonol sulfate; SMS, scymnol sulfate; TCDCA, taurochenodeoxycholic acid; TDHCA, taurodehydrocholic acid. (D) GCDCA (50 $\mu \mathrm{M}, 24$ hours) induced chemokine mRNA expression in human hepatocytes. Data presented as fold change by defining medium control as 1 (mean $\pm \mathrm{SD},{ }^{*} P<0.05$, ${ }^{* *} P<0.01$ vs. Ctrl, $n \geq 5$ ) by 1-way ANOVA.

$B A$-induced liver injury is reduced in $\mathrm{Ccl}^{-1-}$ mice. Because CCL2 is one of the inflammatory cytokines that is elevated in the serum from cholestatic patients, and whose expression is specifically stimulated in our experiments with both human and mouse hepatocytes, we examined the effects of deletion of $\mathrm{Ccl} 2$ in two cholestatic mouse models, i.e., 1\% CA feeding and BDL. As seen in Table 1, plasma alanine aminotransferase (ALT) and BA levels were significantly lower in $\mathrm{CCl2}^{-1-}$ mice after 7 days of $\mathrm{CA}$ feeding as compared with WT controls. Gene expression analysis revealed that deficiency of $\mathrm{Ccl} 2$ also abolished BA-induced mRNA expression of the inflammatory cytokine $\mathrm{Cxcl} 2$ in the liver $\left(\mathrm{Ccl}^{+/+}\right.$with $1 \%$ CA feeding, $3.73 \pm 1.94$ vs. $C_{c l 2^{-/}}$with $1 \%$ CA feeding, $1.29 \pm 0.43$; mean $\pm \mathrm{SD}$, by setting $\mathrm{Ccl}^{+/+}$control diet as $1 ; n=7 ; P<0.05$ ), whereas there were no substantial changes in the expression of BA transporters (e.g., Ntcp, Bsep, Ost $\alpha$, and Mrp4) and Egr1 (data not shown). Since 1\%

CA feeding in mice results in a mild form of liver injury without apparent changes in liver histology, we repeated these experiments in 7-day BDL mice. Again, we detected significantly lower levels of plasma ALT and alkaline phosphatase (ALP) in $C c l 2^{-/-}$BDL mice compared with $C c l 2^{+/+}$BDL mice (Table 1 ), while plasma BA levels were not significantly different. Blinded examination of liver H\&E histology revealed the near absence of necrosis in $C_{c l} 2^{--}$after BDL, whereas multiple necrotic areas were seen in each microscopic field in WT BDL livers (Figure 3A). Hepatic BA levels were also reduced in $C_{c l 2^{-1-}}$ mice compared with WT mice after BDL (Table 1). Liver mRNA expression levels of Cxcl2, Ck19, Cyp7A1, and Ntcp were lower in $C c l 2^{-/}$BDL than in WT BDL mice, whereas there was no significant difference in the expression of Tnfo, Il-1 $\beta$, Egr1, Col1a1, Bsep, and Mrp4 (Supplemental Table 1). Western blots further confirmed that protein expression of $\mathrm{Ck} 19$ and $\mathrm{Ntcp}$ was reduced in $\mathrm{Ccl2}^{-/-}$BDL livers (Supplemental Figure 2A). Furthermore, Western blot analysis of caspase 3 cleavage, an apoptosis indicator, was not detected in either WT or $\mathrm{Ccl}^{-/-}$livers after BDL (Supplemental Figure 2B). 
Table 1. Levels of bile acids in liver and plasma and liver enzymes in plasma in cholestatic mouse models

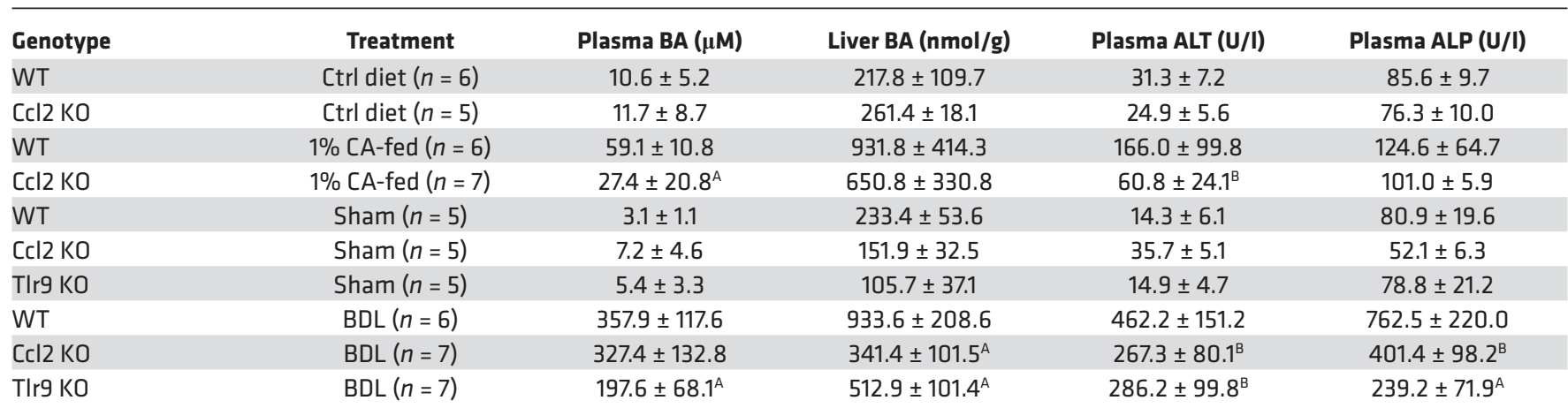

${ }^{A} P<0.01$ and ${ }^{B} P<0.05$ compared with WT treatment controls. ALP, alkaline phosphatase; ALT, alanine aminotransferase; BA, bile acids; BDL, bile duct ligation; CA, cholic acid; Ctrl, control; KO, knockout.

Liver injury is associated with hepatic neutrophil infiltration in BDL mice and cholestatic patients. To gain further insights into how $\mathrm{Ccl} 2$ deficiency protected these livers from BA-induced injury, we analyzed the inflammatory cell profile (neutrophils, NK cells, monocytes/macrophages, and T cells) in the livers of $C c l 2^{-/-}$and WT mice after 7 days of BDL. The number of neutrophils and $\mathrm{T}$ cells in the livers of $\mathrm{Ccl2}{ }^{-/}$BDL mice was significantly lower than in the livers of WT BDL mice, whereas there was no difference in Kupffer cell number in these animals (Figure 3B and Supplemental Figure 3). By contrast, there was no difference in the

A $\mathrm{mHCCl} 2$

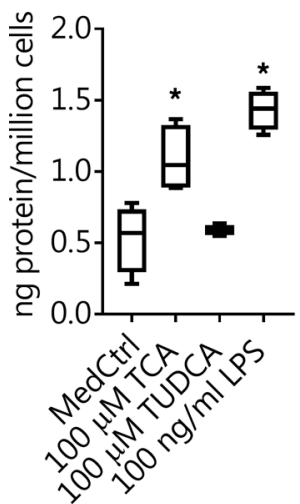

$\mathrm{mNPC} \mathrm{Ccl} 2$

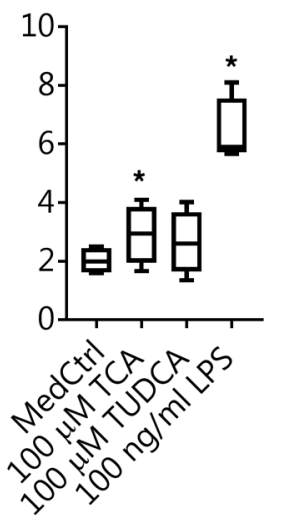

$\mathrm{mNPC} \mathrm{CxCl} 2$

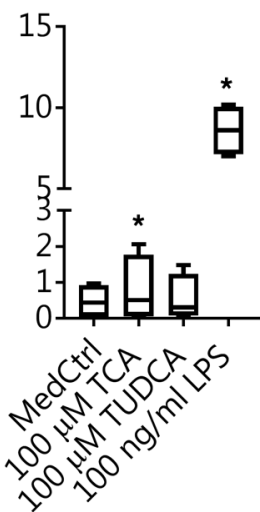

B

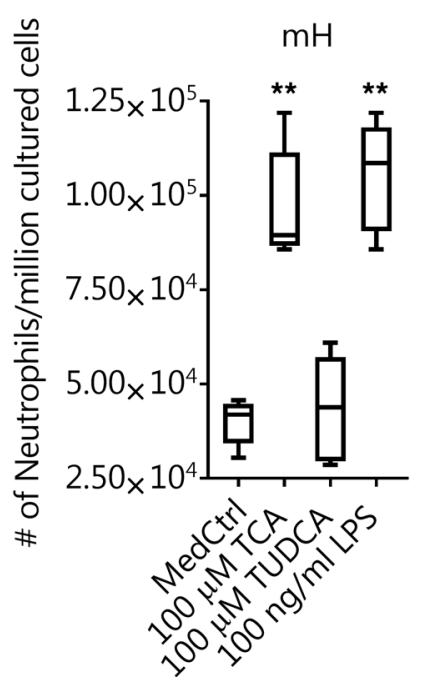

mNPC

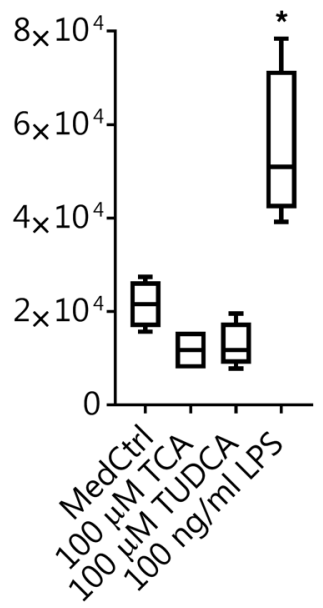

number of neutrophils in the spleen between these 2 groups, although significantly fewer $\mathrm{T}$ cells were detected in the spleen of $\mathrm{Ccl2}^{-1-}$ mice than in WT controls following 7 days of BDL (percentage of $\mathrm{CD}^{+}$cells: WT, $26 \pm 5$ vs. KO, $4 \pm 3$; mean $\pm \mathrm{SD}, n=3, P<0.01)$. Together, these findings confirm that the inflammatory neutrophil response plays an important role in BA-induced liver injury in mice.

To assess the role of neutrophils in patients with cholestatic liver injury, we examined the histology of liver biopsy specimens from 12 cholestatic patients. Levels of serum ALT positively correlated with the degree of periportal but not lobular neutrophil infiltration in these patients (Figure 3C). Since BAs are removed to a greater extent from the circulation by zone 1 periportal

Figure 2. Bile acids stimulate hepatocyte release of proinflammatory cytokines that function as chemoattractants for neutrophils. (A) Elevated protein levels of $\mathrm{C} \mathrm{Cl} 2$ and $\mathrm{Cxc} / 2$ were detected in the media of taurocholic acid-treated (TCA-treated) mouse hepatocytes $(\mathrm{mHs})$ but not from mouse nonparenchymal cells (mNPCs) after a 24-hour treatment. ${ }^{*} P<0.05$ vs. medium control (MedCtrl), $n=4$. (B) Culture medium from TCA-treated hepatocytes but not NPC-stimulated migration of mouse neutrophils across transwell membranes. TUDCA, tauroursodeoxycholic acid. ${ }^{*} P<0.05,{ }^{* *} P<$ 0.01 vs. MedCtrl, $n \geq 4$ by 1-way ANOVA. 
A
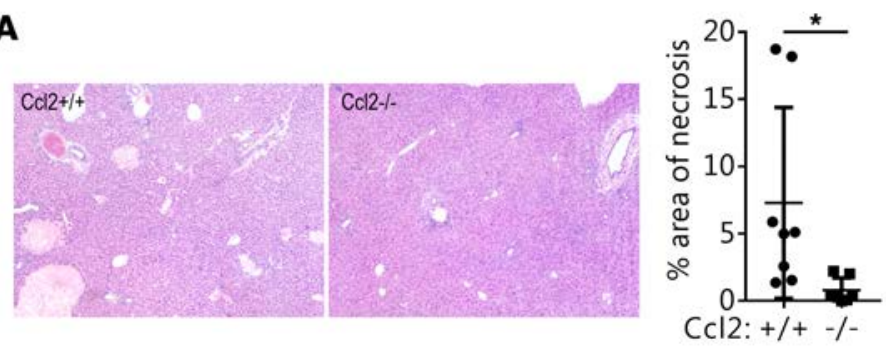

B
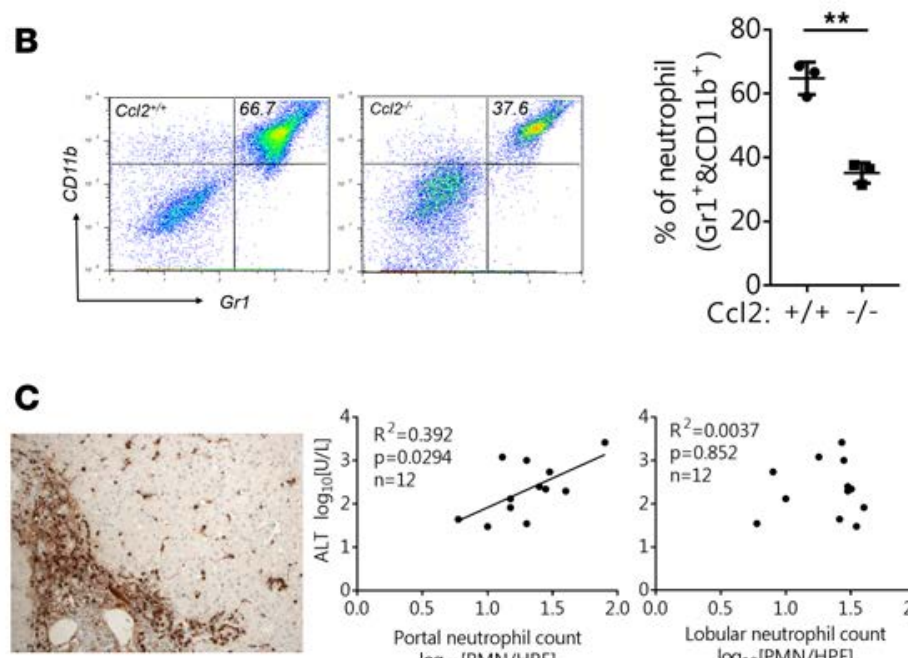

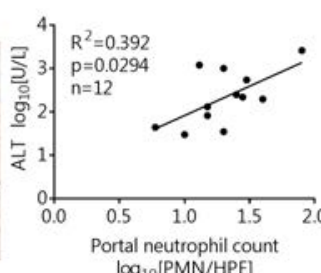

$\log _{10}[\mathrm{PMN} / \mathrm{HPF}]$

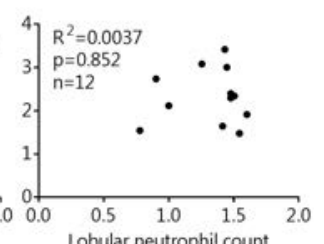

$\log _{10}[\mathrm{PMN} / \mathrm{HPF}]$
Figure 3. Inflammatory cells play an important role in cholestatic liver injury in both mice and humans. (A) Representative H\&Estained liver sections from $\mathrm{Ccl}^{+{ }^{++}}$and $\mathrm{Ccl}^{-2^{--}}$mice after bile duct ligation (BDL) for 7 days (left). Hepatocyte necrosis is significantly reduced in $\mathrm{Ccl}^{-1-}$ mice after 7 days of $\mathrm{BDL}$ (right), as assessed by quantitative analysis of liver sections (mean $\pm \mathrm{SD},{ }^{*} P<0.05, n=$ 6-7). Original magnification, $\times 40$. (B) A pair of representative flow cytometry plots of neutrophils (CD11 b+ and $\mathrm{Cr}^{+}{ }^{+}$cells) in $\mathrm{Ccl} 2^{+/+}$and $C \mathrm{Cl} 2^{-1-}$ livers after $\mathrm{BDL}$ is on the left. Neutrophils were significantly reduced (right) in livers of $\mathrm{Ccl}^{-1-}$ mice after 7 -day BDL. ${ }^{* *} P<0.01$, $n=3$. (C) Immunoperoxidase stain (left) obtained from a representative cholestatic patient liver biopsy showing many portal and lobular myeloperoxidase-positive cells (original magnification, $\times 200$ ). Hepatic injury (serum alanine aminotransferase [ALT]) positively correlated with periportal (middle) but not lobular (right) neutrophil infiltration in the liver of cholestatic patients. Statistical significance in $\mathbf{A}$ and $\mathbf{B}$ determined by 2-tailed Student's t test.

hepatocytes, these findings are consistent with a specific role for BA-induced inflammatory response in the pathogenesis of human cholestatic liver injury.

BA-stimulated chemokine expression in hepatocytes is mediated by NTCP. To better understand why BA-stimulated cytokine production was hepatocyte specific, we speculated that the hepatocyte BA transporter, Ntcp (S1c10a1), may be required to mediate this event owing to its robust uptake of conjugated BA $(20,21)$. As shown in Figure 4A, when Ntcp expression in mouse hepatocytes was knocked down by siRNAs, Cxcl2 induction by TCA was significantly diminished. To determine whether pharmacological inhibition of Ntcp would achieve the same effect, we first synthesized 2 BA analogs by conjugating biotin to CA and ursodeoxycholic acid (UDCA). ${ }^{3} \mathrm{H}$-TCA uptake assays in mouse hepatocytes confirmed that these 2 compounds blocked Ntcp transport activity (Supplemental Figure 4). When we applied these potentially novel Ntcp inhibitors to mouse hepatocytes, both compounds significantly reduced TCA and glycocholic acid (GCA) induction of Cxcl2 mRNA expression (Figure 4B). These findings indicate that BAs must be taken up by the hepatocyte and that accumulation of intracellular BAs is an absolute requirement to stimulate this inflammatory cytokine response. These studies also explain why hepatocytes, but not the other NPCs, are specifically vulnerable to BAs since only hepatocytes express NTCP/Ntcp.

$B A s$ cause mitochondrial injury and ER stress but without changing ROS production in mouse hepatocytes. To elucidate the intracellular signaling pathway that results in this pattern of cytokine expression, we examined whether BAs induced markers of mitochondrial injury, ER stress, and ROS. A JC-1 assay demonstrated that endogenous BAs (i.e., TCA, GCA, and GCDCA) at pathophysiologic concentrations caused mitochondrial membrane potential changes in mouse hepatocytes (Supplemental Figure 5, A and B). Western blot

Figure 4. Bile acid induction of $\mathrm{Cxcl} 2$ in mouse hepatocytes is reduced after inhibition of the bile acid transporter Ntcp. (A) Ntcp knockdown reduced taurocholic acid (TCA) induction of Cxcl2 mRNA expression. Forty-eight hours after siRNA transfection, cells were treated with TCA for an additional 24 hours. Results are the mean $\pm \mathrm{SD}, n=4 .{ }^{*} P<0.05$ by 1 -way ANOVA. (B) Biotinylated cholic acid (BCA) and ursodeoxycholic acid (BUDCA) reduced TCA and glycocholic acid (CCA) induction of Cxcl2. Cells were treated with the indicated inhibitors for 6 hours (mean $\pm S D, n=4$ ). ${ }^{*} P<0.05$ by 1-way ANOVA.
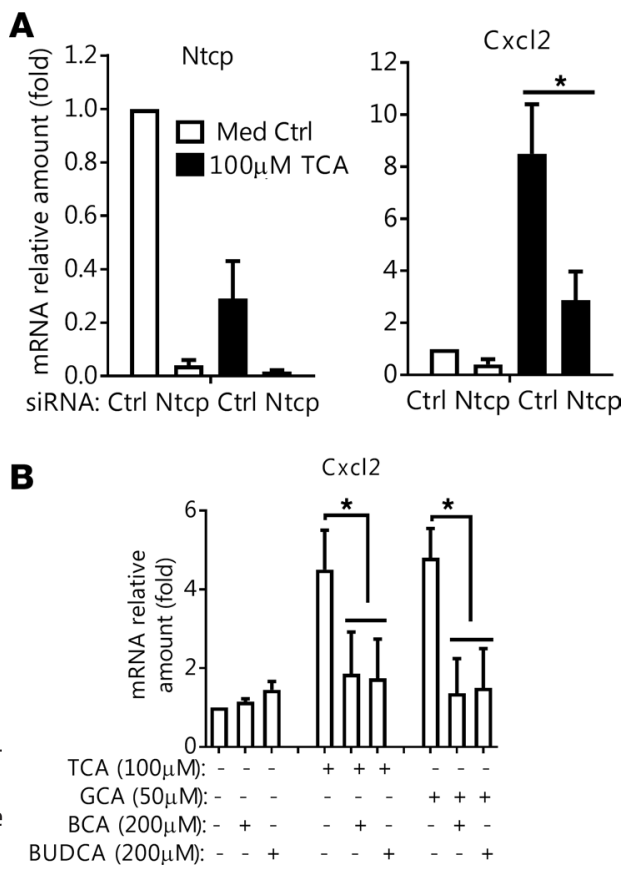
A

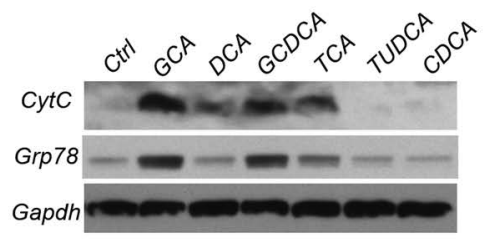

D

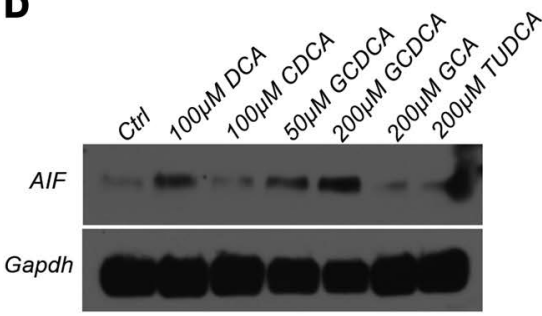

B

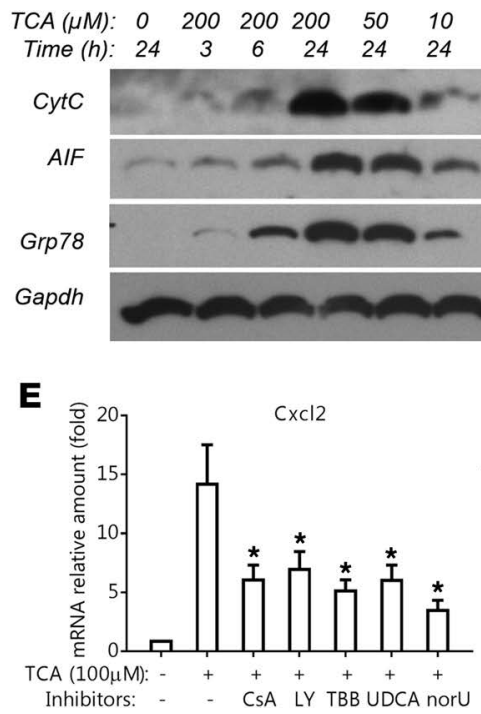

C
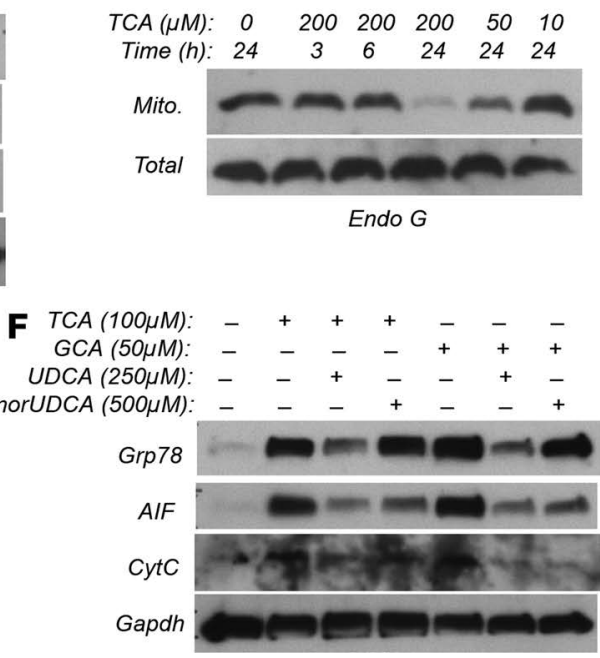

Figure 5. Bile acids induce hepatocyte mitochondrial injury and ER stress. (A) Representative Western blot detecting leakage of mitochondrial protein cytochrome $c$ (CytC) and ER protein Grp78 into the cytosol in mouse hepatocytes after a 24-hour treatment with chenodeoxycholic acid (CDCA, $50 \mu \mathrm{M})$, glycocholic acid (GCA, $200 \mu \mathrm{M}$ ), glycochenodeoxycholic acid (CCDCA, $200 \mu \mathrm{M}$ ), deoxycholic acid (DCA, $50 \mu \mathrm{M}$ ), taurocholic acid (TCA, $200 \mu \mathrm{M})$, and tauroursodeoxycholic acid (TUDCA, $200 \mu \mathrm{M}$ ). (B) Representative Western blot demonstrating that TCA treatment released CytC, apoptosis-inducing factor (AIF, a mitochondria-specific protein), and Grp78 from mitochondria and the ER into the cytosol in a dose- and time-dependent manner in mouse hepatocytes. (C) Representative Western blot showing reduction of mitochondrial (Mito) but not total cellular endonuclease $G$ (Endo G) protein expression in TCA-treated mouse hepatocytes (dose and time dependency). (D) Western blot detection of GCDCA treatment (24 hours) resulting in AIF leaking into the cytosol in human hepatocytes. (E) TCA $(100 \mu \mathrm{M})$ induction of Cxcl2 mRNA expression in mouse hepatocytes was significantly reduced by cyclosporin A (CsA, $3 \mu \mathrm{M})$, LY294002 (LY, $40 \mu \mathrm{M}$ ), 4,5,6,7-tetrabromobenzotriazole (TBB, $25 \mu \mathrm{M}$ ), ursodeoxycholic acid (UDCA, $250 \mu \mathrm{M}$ ), and norUDCA (norU, $500 \mu \mathrm{M})$. Cells were treated for 24 hours (mean $\pm S D, n \geq 4$ ). ${ }^{*} P<0.05$ vs. TCA treatment alone by 1 -way ANOVA. (F) UDCA and norUDCA treatment reduced bile acid-induced leakage of AIF and Grp78 protein into cytosol in mouse hepatocytes. All Western blots shown here have been repeated in at least 3 independent experiments.

analysis detected mitochondria-specific protein cytochrome $c$, and ER-specific protein Grp78 in the cytosolic fraction (Figure 5A) after treatment with GCA, TCA, GCDCA, and deoxycholic acid (DCA), but not TUDCA or CDCA. Figure 5B demonstrates that TCA's deleterious effects on mitochondria and the ER were both dose- and time-dependent as evidenced by cytochrome $c$, apoptosis-inducing factor (AIF), and Grp78 leakage into the cytosol, which positively correlated with BA induction of Ccl2 and Cxcl2 mRNA expression in these mouse hepatocytes (Figure 1B). A similar dose and time dependency was also seen with mitochondrial endonuclease $\mathrm{G}$ protein expression (Figure $5 \mathrm{C}$ ). Furthermore, as seen in mouse hepatocytes, the leakage of AIF from mitochondria to the cytosol was also detected in GCDCAtreated human hepatocytes (Figure 5D). Of particular note, AIF and endonuclease $G$ are nucleases that are able to cleave and fragmentize DNA. In contrast, activation of caspase 3 was not detected, as BA treatments did not lead to its cleavage in hepatocytes from both humans and mice (Supplemental Figure 6), in agreement with earlier reports $(7,8)$. Furthermore, blinded assessment of propidium iodidestained mouse hepatocytes did not reveal any difference between TCA (100 $\mu \mathrm{M}$ for 24 hours) treatment and its control. Finally, increased mRNA expression of the ER markers Grp78 and Xbp1 was detected in TCA-treated mouse hepatocytes. In contrast, ROS were not detected in these cells when analyzed by MitoSOX and 2', $7^{\prime}$-dichlorofluorescein diacetate assays (Supplemental Figure 5C and data not shown).

To further determine whether BA-induced mitochondrial injury is the cause of cytokine induction, and also to identify signaling pathways that may be involved in the process, we treated mouse hepatocytes with cyclosporine A (CsA; a known inhibitor of mitochondrial permeability transition), UDCA (the current FDA-approved standard treatment for cholestasis), norUDCA (a UDCA derivative showing hepatoprotective effects in cholestatic rodents and currently in phase II clinical trial for primary sclerosing cholangitis), LY294002 (a PI3K inhibitor), 4,5,6,7-tetrabromobenzotriazole (TBB; a casein kinase 2 inhibitor), necroptosis inhibitors, MAPK inhibitors, and antioxidants. As shown in Figure 5E, $3 \mu \mathrm{M}$ CsA, $40 \mu \mathrm{M}$ LY294002, and $25 \mu \mathrm{M}$ TBB significantly reduced TCA induction of Cxc12, whereas inhibitors of necroptosis (necrostatin-1 and necrosulfonamide, up to 20 and $5 \mu \mathrm{M}$, respectively), MAPKs (10 $\mu \mathrm{M}$ U0126, $10 \mu \mathrm{M}$ SP600125, or 10 $\mu \mathrm{M}$ SB203580), and EGFR (5 $\mu \mathrm{M}$ AG1478) did not exert the same effect (data not shown). Furthermore, 
A
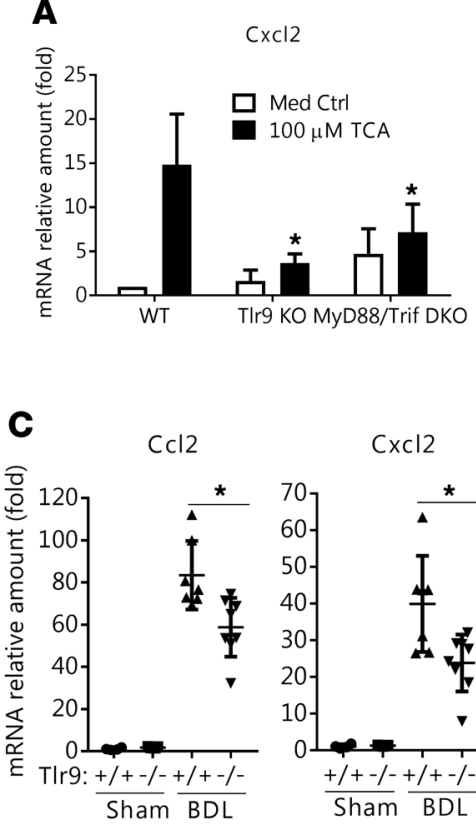

B
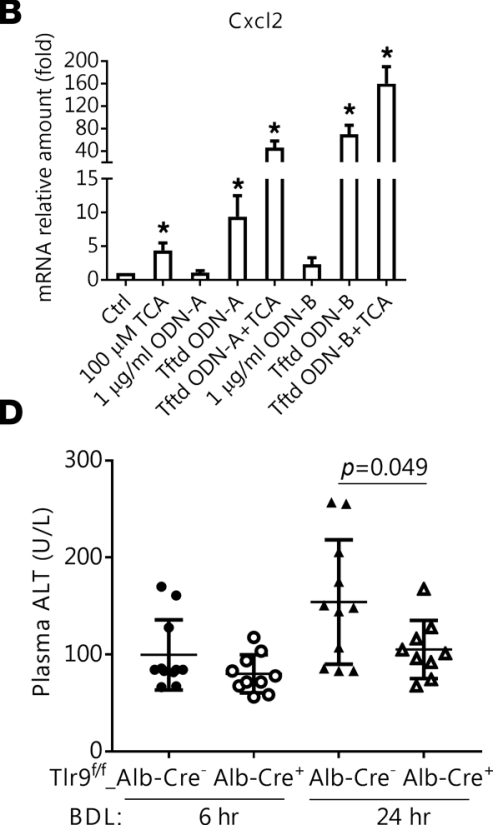

Figure 6. Tlr9 is involved in bile acid induction of $\mathrm{Cxcl} 2$ in vitro in mouse hepatocytes and in vivo in BDL mouse liver. (A) Taurocholic acid (TCA, $100 \mu \mathrm{M})$ stimulation of Cxcl2 mRNA expression was significantly decreased in hepatocytes from TIr9 knockout (KO) and MyD88/Trif double-knockout (DKO) mice when compared with hepatocytes from WT mice. Cells were treated for 24 hours, and results are the mean $\pm \mathrm{SD},{ }^{*} P<0.05$ vs. WT TCA treatment, $n \geq 4$. (B) Transfected (Tftd) TIrg agonists (ODN-A and ODN-B, $1 \mu \mathrm{g} / \mathrm{ml}$ ) showed synergistic effects with TCA (100 $\mu \mathrm{M})$ in stimulation of Cxcl2 mRNA expression in mouse hepatocytes. Sixteen hours after transfection, cells were treated with TCA for 6 hours (mean \pm SD, $n \geq 3$ ). The control cells were treated with transfection medium, and their Cxcl2 expression was set to 1 . (C) C $\mathrm{Cl} 2$ and Cxcl2 liver mRNA expression was significantly lower in Tlr9 whole-body KO mice than in WT mice after 7 days of bile duct ligation (BDL). Results are the mean $\pm S D,{ }^{*} P<0.05$, $n=6-7$. (D) Alanine aminotransferase (ALT) serum levels were significantly reduced in hepatocyte-specific TIr9 KO

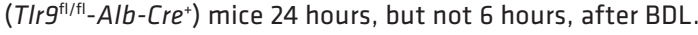
$P$ values determined by 2-tailed Student's $t$ test.

antioxidants ( $2 \mathrm{mM} N$-acetylcysteine, $1 \mu \mathrm{M}$ MitoQ) and pan caspase inhibitor z-VAD-FMK $(20 \mu \mathrm{M})$ did not substantially block TCA-induced Cxcl 2 mRNA expression (data not shown). In contrast, both UDCA (250 $\mu \mathrm{M})$ and norUDCA $(500 \mu \mathrm{M})$ significantly reduced TCA stimulation of Cxcl 2 mRNA expression (Figure $5 \mathrm{E})$. Interestingly, norUDCA blocked translocation of AIF from mitochondria to the cytosol after BA treatment, whereas UDCA reduced Grp78 leakage from the ER (Figure 5F).

TCA induction of CXcl2 is diminished in Tlr9 KO mouse hepatocytes. Since our findings indicated that BAs produced mitochondrial injury and released nucleases capable of fragmentizing DNA, we examined the role of Tlr9, which is activated by intracellular DNA. When $\operatorname{Tl} 9^{-1-}$ mouse hepatocytes were treated with TCA, significantly less induction of $\mathrm{Cxcl} 2$ was detected compared with WT hepatocytes (Figure 6A). The involvement of Tlr9 in TCA induction of Cxcl2 was further confirmed in MyD88/Trif double-KO (DKO) mouse hepatocytes (Figure 6A). Of note, MyD88 and Trif are downstream signaling molecules of Tlr9. Furthermore, transfecting mouse hepatocytes with synthetic Tlr9 ligand CpG ODNs stimulated Cxc12 expression, and addition of BAs showed synergistic effects (Figure 6B), supporting Tlr9 involvement in $\mathrm{BA}$ induction of $\mathrm{Cxcl} 2$. However, in contrast to immune cells, hepatocytes are not affected by extracellular DNA or $\mathrm{CpG}$ ODNs, because directly adding them to the cell culture did not result in stimulation of $\mathrm{Cxc12}$ expression (Figure $6 \mathrm{~B}$ ). NFкB is also unlikely to be involved in this signaling pathway, as BAs did not stimulate a Tlr9-NFאB reporter gene using an HEK-blue reporter assay, after these cells were first transfected with NTCP to allow BA entry (data not shown). BAs also did not stimulate an NFкB reporter construct in transfected mouse hepatocytes (data not shown), in agreement with an earlier report (22).

Reduced liver injury in both conventional and hepatocyte-specific Tlr9 KO mice after BDL. Finally, to verify Tlr9's role in cholestatic liver injury in vivo, we carried out BDL in 2 strains of $\operatorname{Tl} 9 \mathrm{KO}$ mice, i.e., conventional whole-body and hepatocyte-specific. First, we assessed liver injury in Tlr9 conventional KO (Tlr $9^{--}$) mice after BDL for 7 days. Compared with WT BDL controls, plasma ALT, ALP, and BA levels were significantly lower in $T 7 \mathrm{r}^{-/-}$BDL mice, indicating less liver injury in these mice (Table 1). BA concentrations were also lower in the livers of these mice (Table 1). Examination of liver H\&E histology and immunohistochemical labeling of Ck19 revealed significantly less bile duct proliferation in these mice than in WT controls after BDL. This was further confirmed by detecting hepatic expression of Ck19 mRNA and protein (Supplemental Figure 7). Furthermore, significantly less mRNA expression of $\mathrm{Ccl} 2$ and $\mathrm{Cxcl} 2$ was also detected in $\mathrm{Tlr}^{-/-}$livers than in the WT controls after BDL (Figure 6C). However, there were no significant differences between these 2 groups in liver expression of Egr1 and BA transporters, including Bsep, Mrp2, Mrp4, and Ntcp.

To further test the role of hepatocyte Tlr9 in the BA-initiated inflammatory response, we performed shortterm (24 hours) BDL in hepatocyte-specific Tlr9 $\mathrm{KO}\left(\mathrm{Tlr} \mathrm{g}^{\mathrm{q} / \mathrm{fl}} / \mathrm{Alb}-\mathrm{Cre}^{+}\right)$mice in order to catch the early events in BA-induced liver injury. Analyses of plasma levels of ALT, ALP, and BA 6 hours and 24 hours after BDL 


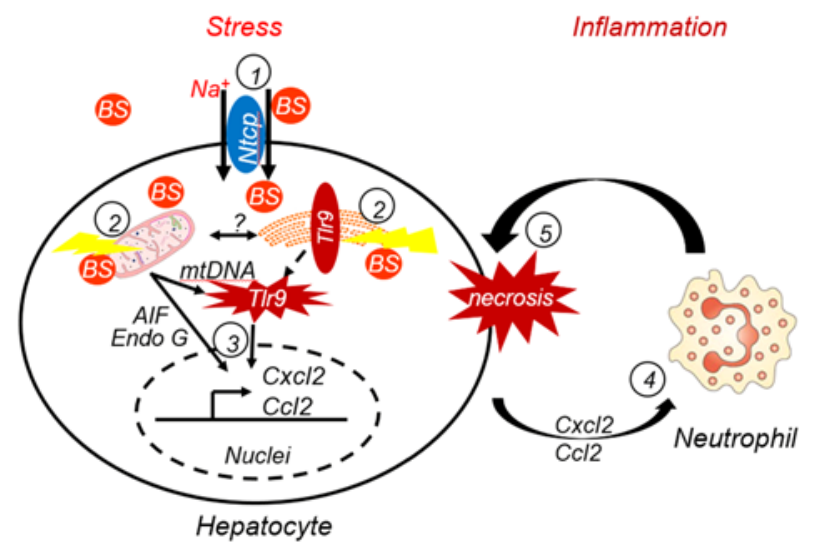

Figure 7. A model representing the mechanism of bile acid-induced liver injury as described in this study. (1) Bile salts (BS, conjugated bile acids) must be first taken up by transporter Ntcp and accumulate in hepatocytes. (2) Elevated levels of BS in hepatocytes cause ER stress and mitochondrial damage, resulting in release of mitochondrial DNA (mtDNA) and proteins. Cytosolic mtDNA in turn triggers an innate immune response by activating TIr9-dependent and possibly other independent signaling pathways, resulting in (3) stimulation of inflammatory chemokine expression (e.g., C $\mathrm{Cl} 2$ and $\mathrm{Cxcl2}$ ). (4) Hepatocytereleased chemokines recruit neutrophils, and (5) the neutrophil-mediated inflammatory response results in hepatocyte necrosis.

revealed significantly less ALT in $T / r 9^{\natural / / 1} / \mathrm{Alb}_{-} \mathrm{Cre}^{+}$mice at the 24-hour time point when compared with their littermate WT controls (Figure 6D), whereas there were no differences in ALT, ALP, and BA at the 6-hour time point, nor in ALP and BA at the 24-hour time point (data not shown). Examination of H\&E histology and immunohistochemical labeling of $\mathrm{Ly} 6 \mathrm{G}^{+}$cells from liver tissue indicated that on average there was less necrosis and neutrophil infiltration in $\mathrm{Tl} \mathrm{g}^{\mathrm{a} / \mathrm{fl}} / \mathrm{Alb}-\mathrm{Cre}^{+}$mice, but the differences did not reach statistical significance due to large variation among animals in this short-term experiment (data not shown). Together, these in vivo findings confirm a significant role for Tlr9 in BA-induced liver injury.

\section{Discussion}

During cholestasis, serum and liver BA levels are elevated and result in hepatic injury. Because the pathogenesis of BA-induced liver injury remains elusive, the development of new therapies for treating cholestasis has been limited. In this report, we provide new insights into the mechanism of BA-induced liver injury that have potentially novel therapeutic implications. We found that hepatocytes but not other liver cells released inflammatory chemoattractants and caused neutrophil chemotaxis when BA concentrations were at pathophysiological levels. This cell-type-specific event was mediated through Ntcp, the hepatocyte-specific sodium-dependent conjugated-BA uptake transporter. The accumulation of intracellular BA resulted in mitochondrial injury and ER stress, and triggered an innate immune response by activating Tlr9, thereby initiating an inflammatory response where neutrophils appear to play a central role in the liver injury, as illustrated in Figure 7.

This model is supported by a number of observations. First, in agreement with previous reports $(6,7)$, apoptotic markers were not detected in the livers of our cholestatic mouse models nor in vitro in hepatocytes from humans or mice after treatment with endogenous BAs at pathophysiological levels (25-200 $\mu \mathrm{M})$ (Supplemental Figures $2 \mathrm{~B}$ and 6). These findings challenge the theory that BA injures the liver in cholestasis solely by a direct cytotoxic effect.

Second, endogenous BAs stimulated the expression of chemoattractants $\mathrm{Ccl} 2$ and $\mathrm{Cxcl} 2$ in mouse hepatocytes in both a dose- and time-dependent manner (Figure 1). TCA significantly increased Cxc12 expression in mouse hepatocytes even at concentrations as low as $25 \mu \mathrm{M}$, a pathophysiologically relevant serum level seen in both cholestatic patients and animals. We also demonstrated that chemokines released by hepatocytes were able to recruit neutrophils (Figure 2). These findings emphasize the relevance of hepatocyte-generated chemoattractant expression as an early initiating event in the pathogenesis of the inflammatory response during cholestasis. Because the FXR-specific agonist GW4064 and TUDCA did not induce $\mathrm{Ccl} 2$ and $\mathrm{Cxcl} 2$ expression as did other endogenous BAs in our hepatocyte cultures (Figure 1C), and also because BA induction of chemokines in mouse hepatocytes is FXR independent (11), we conclude that this induction is not mediated through the BA nuclear receptor FXR, but rather through other unidentified signaling pathways. Most importantly, we verified that GCDCA at pathophysiologically relevant concentrations injured mitochondria and stimulated inflammatory chemoattractant expression in human hepatocytes, (Figures 1D and 5D), indicating that humans and mice share analogous mechanisms in BA-induced cholestatic liver injury.

Third, NTCP plays a critical role in the pathogenesis of cholestatic liver injury since it is required for conjugated BA to be taken up into hepatocytes. Of note, more than $95 \%$ of BAs are in conjugated form in 
sera from cholestatic patients as well as sera of mice after 14-day BDL $(6,23)$. Normally, once BAs get into hepatocytes, they are rapidly excreted into the lumen of the bile canaliculus. However, during cholestasis, BA excretion is restricted, resulting in the accumulation of BAs in hepatocytes. Since NTCP is expressed only in hepatocytes, this provides an explanation as to why BAs did not induce inflammatory cytokines in liver NPCs. Indeed, in the present study, BA uptake was diminished when Ntcp was inhibited, and resulted in less induction of Cxcl2 (Figure 4). Recently, a rare case of NTCP deficiency was described in which the patient was completely protected from cholestatic liver injury, despite extremely high levels of BAs in the blood, consistent with our findings (20). These observations also suggest that pharmacological inhibition of NTCP might be a novel therapy for patients with cholestasis.

Fourth, BA induction of $\mathrm{Cxc1} 2$ was diminished in $\mathrm{Tlr}^{-/-}$hepatocytes (Figure 6A), indicating that hepatocyte Tlr9 plays an important role in initiating hepatic inflammation. This is further supported by the observations that Cxc12 induction was significantly reduced in MyD88/Trif DKO mouse hepatocytes by BAs as well as the synergistic effects of TCA and transfected CpG ODNs on Cxc12 expression (Figure 6B). Most importantly, reduced liver injury in both conventional and hepatocyte-specific Tlr 9 KO BDL livers further confirmed that Tlr9 plays a role in the pathogenesis of cholestatic liver injury (Table 1, Figure 6, and Supplemental Figure 7). This conclusion is also consistent with an earlier report in which reduced liver fibrosis and $\mathrm{Ccl} 2$ expression were detected in conventional Tlr $9^{-/-}$mice after BDL for 3 weeks (24). However, because directly adding DNA (up to $50 \mu \mathrm{g} / \mathrm{ml}$ ) or CpG ODNs (a Tlr9 agonist) in mouse hepatocyte culture did not stimulate Cxc12 expression (Figure 6B and data not shown), we speculate that hepatocytes do not respond to extracellular DNA, in contrast to immune cells. It seems likely that Tlr9 is activated by endogenous intracellular DNA (e.g., mtDNA) in mouse hepatocytes, since markers of mitochondrial injury were observed in these cells after BA treatment (Figure 5 and Supplemental Figure 5) and BA induction of $\mathrm{Cxcl} 2$ in these cells was diminished when the mitochondrial membrane potential was protected with CsA (Figure 5E). BA-induced deleterious effects in mitochondria have been reported in NTCP-transfected HepG2 cells and rat hepatocytes $(25,26)$. Interestingly, norUDCA treatment prevented AIF leakage from mitochondria and reduced Cxcl2 induction, providing a mechanistic explanation for the beneficial effect of this drug in cholestatic rodent models $(5,27,28)$. However, it remains to be elucidated how intracellular DNA activates Tlr9, which normally resides on the ER and endosomes. One possibility requiring further study is that injured mitochondria undergo autophagy/mitophagy and present mtDNA to Tlr9 (29).

Because BA induction of Cxcl2 expression was not completely abolished in $T / r 9^{-1-}$ hepatocytes, we speculate that there are also Tlr9-independent signaling pathways involved in this regulation. This also explains why liver injury was reduced but not abolished in $T / r 9^{-1-}$ BDL mice. Additional pathways may include other intracellular DNA sensors in hepatocytes and the transcription factor Egr1. It has been shown that Egr1 plays an important role in BA stimulation of the expression of certain chemokines in mouse hepatocytes and in cholestatic liver injury in BDL mice $(11,30)$. It is likely that Tlr9 signaling is independent of Egr1 activity because there was no difference in liver Egr1 mRNA expression between Tlr9 ${ }^{-/-}$mice and the WT control group after BDL. In addition, our data suggest that PI3K and casein kinase 2 are involved in BA induction of $\mathrm{Cxcl} 2$ in mouse hepatocytes because their specific inhibitors reduced BA's stimulatory effects (Figure 5E). The PI3K involvement is consistent with an early report (16). However, in contrast to this report, we did not detect substantial differences when MAPK inhibitors were applied to the cells. These apparent discrepancies may be due to differences in the doses of TCA and inhibitors, and most likely the differences in treatment times. Our cells were treated with BA for 24 hours in collagen-sandwich culture to mimic a chronic cholestatic condition, whereas the earlier report treated the cells only for 0.5 hours. We also stress that the results of our study do not exclude a role for other NPCs in the overall inflammatory response as cholestasis progresses, but rather emphasize the pivotal role that the hepatocyte plays in the initiation of this response.

Finally, our studies provide additional support for the hypothesis that cholestatic hepatocyte death is due to the inflammatory neutrophil response and the oxidative cell injury that this produces (23). As demonstrated in this report, the hepatocyte that is injured by BA first sends out signaling molecules, e.g., chemoattractants, to recruit immune cells to initiate a resolve-or-heal response similar to other forms of liver injury (31). It is likely that neutrophils, and perhaps $\mathrm{T}$ cells, are responsible for killing the injured hepatocytes during cholestasis, because immune-cell profiling revealed significantly fewer neutrophils and $\mathrm{T}$ cells in $\mathrm{Ccl2}^{-/-}$BDL livers where lower plasma ALT levels were also detected (Figure 3, Supplemental Figure 3, 
and Table 1). This speculation is also supported by previous reports $(10,16)$. Importantly, hepatic injury in cholestatic patients positively correlated with the degree of periportal neutrophil infiltration (Figure 3C), confirming a role for inflammation in the pathogenesis of cholestatic liver injury in humans. Kupffer cells did not seem to play a role in injuring hepatocytes in these cholestatic models, as there were no differences in macrophage content between WT and $C_{c l} 2^{-/-}$after BDL. Others have shown that depletion of Kupffer cells does not reduce liver injury in BDL mice (32).

In summary, the findings in our studies reveal several potentially novel mechanisms that underlie the pathogenesis of cholestatic liver injury (Figure 7) and that may serve as potential targets for therapeutic intervention in clinical cholestatic disorders. By blocking the hepatic uptake transporter, NTCP, BA levels in hepatocytes may be diminished and tissue injury reduced. Protecting mitochondria from the deleterious effects of BAs or inhibiting Tlr9 should diminish the release of inflammatory cytokines, which in themselves may be targeted by blocking their receptors. Combination of such agents would be predicted to have synergistic protective effects. Future clinical studies will be needed to test these hypotheses.

\section{Methods}

Materials. Chemicals were purchased from Sigma-Aldrich, except where otherwise specified. Cell culture media (DMEM and Williams' E), FBS, penicillin/streptomycin, trypsin, PBS, and Lipofectamine RNAiMax were from Life Technologies. Collagen-coated plates and collagen were purchased from BD Biosciences. ECL reagents were from Thermo Scientific. norUDCA was a gift from Alan Hofmann (UCSD, San Diego, California, USA). Mouse Ntcp (Slc10a1) siRNA oligos (siGENOME SMARTpool) and control siRNAs were purchase from Dharmacon. ODN-A (ODN 1585) and ODN-B (ODN D-SL01) were

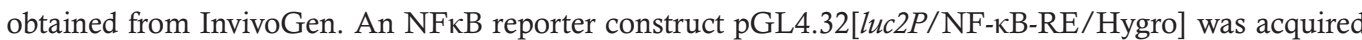
from Promega. Metafectene Pro was from Biontex Laboratories $\mathrm{GmbH}$. MitoQ was a gift from Michael Murphy (MRC Mitochondrial Biology Unit, Cambridge, UK). SB203580, U0126, SP600125, and AG1478 were purchased from EMD Millipore. Mouse $\mathrm{Ccl} 2$ and $\mathrm{Cxcl} 2$ protein quantification ELISA kits were purchased from RayBiotech and R\&D Systems, respectively.

Synthesis of biotinylated CA and UDCA. CA and UDCA were conjugated with biotin at their carboxyl terminus. Because these BA conjugates have a long bulky side chain compared with their endogenous counterparts, they can inhibit the uptake of BA. The structure and syntheses procedures are described in Supplemental materials.

Animals. Whole-body gene KO Ccl2 ${ }^{-/-}$and Tlr $9^{-/-}$and $M y D 88^{-1-} /$ Trif $^{\prime-}$ mice are on the C57BL/6 background. $C \mathrm{Cl} 2^{-/-}$breeding pairs were from Themis R. Kyriakides (Yale University, New Haven, Connecticut, USA). Tlr9 hepatocyte-specific KO mice were generated by breeding Alb-Cre mice with Tlr fl/fl $^{\text {mice, }}$, and offspring $T \mathrm{Tr}^{\mathrm{A} / \mathrm{fl}} / \mathrm{Alb} \mathrm{Cr} \mathrm{C}^{+}$were used for the experiments. The details of $\mathrm{Tl} \mathrm{r}^{\mathrm{A} / \mathrm{fl}}$ creation were published

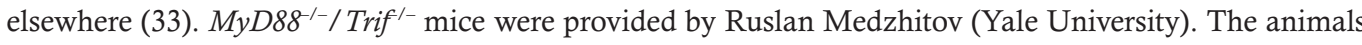
were kept in a pathogen-free environment on a controlled 12-hour light/dark regime in the animal facility of Yale University. Only 10- to 13-week-old males were used in this study. For CA-feeding-induced liver injury, both $\mathrm{Ccl2}^{-1-}$ and WT mice were randomly separated into 2 groups. Each group was fed either the control diet, composed of standard rodent chow, or the BA diet, composed of standard chow with $1 \%$ CA (wt/wt), for 7 days. For obstructive cholestasis-induced liver injury, mice were subjected to BDL or sham operation for 7 days. To collect tissues, mice were fasted overnight before sacrifice. Plasma and liver were collected, and analyzed as previously described (34).

Mouse hepatocyte and NPC preparation and treatment. Both hepatocytes and NPCs were isolated from 10 - to 20-week-old mice using collagenase perfusion as previously described (35). Hepatocytes were maintained in collagen-sandwich culture to closely resemble in vivo conditions. NPCs contained liver endothelial, stellate, and Kupffer cells; all were maintained in DMEM containing 10\% FBS. Mouse macrophages were isolated and maintained as previously described (36). Mouse primary cholangiocytes were provided by Carlo Spirli (Yale University) as previously described (37). All cell cultures were treated with indicated chemicals and collected within 96 hours after isolation. Protein and mRNA expression were detected as previously described (34). Antibodies against cytochrome $c$ (catalog 4272S), AIF (catalog 4642S), endonuclease G (catalog 4969S), and caspase 3 (catalog 9665S) were from Cell Signaling Technology. Grp78 antibody (catalog 610978) was from BD Transduction Laboratories. To transfect mouse hepatocytes, siRNA or oligo DNA were mixed with Lipofectamine RNAiMax in Opti-MEM (Life Technologies) following the manufacturer's instructions and added to the culture medium 3 hours after the cells were plated on 
collagen-coated plates $\left(4 \times 10^{5}\right.$ cells/well in a 12 -well plate). For NFkB reporter assays, the reporter construct or the empty-vector control DNA were mixed with Metafectene Pro following the manufacturer's instruction and added to mouse hepatocytes seeded in 24 -well plates $\left(2 \times 10^{5}\right.$ cells/well). The transfected cells were overlaid with collagen 24 hours after transfection. Twenty-four hours later, the cells were treated with the indicated concentrations of BA for the indicated times.

Neutrophil transwell migration. Mouse neutrophils were prepared and transwell migration experiments were performed as previously described in 24-well Transwell plates (Permeable Supports 6.5-mm insert, $3.0-\mu \mathrm{m}$ polycarbonate membrane provided by Costar) $(38,39)$. Briefly, $0.5 \mathrm{ml} /$ well of 24 -hour cell culture medium was placed in a 24 -well plate and $0.1 \mathrm{ml}$ of $2 \times 10^{6}$ neutrophils $/ \mathrm{ml}$ were loaded into the insert chamber, and incubated at $37^{\circ} \mathrm{C}$ for 100 minutes. After incubation, the insert chamber was removed, and migrated cells were fixed with $4 \%$ paraformaldehyde and counted under a microscope. Migrated neutrophil number was normalized to the initial number of hepatocytes or NPCs from which the culture medium was collected.

Human hepatocytes. Human hepatocytes were obtained through the Liver Tissue Cell Distribution System, which was funded by NIH contract N01-DK-7-0004/HHSN267200700004C. The cells were maintained as previously reported (35).

Human cholestatic liver. Twelve examples of cholestatic liver injury (Supplemental Table 2) were randomly selected from the pathology database. H\&E-stained slides for each liver biopsy were reviewed prior to inclusion in the study. Specimens from patients with bacterial infections, liver transplant, or a diagnosis of malignancy were excluded. The paraffin-embedded formalin-fixed liver biopsies were stained with antibody against myeloperoxidase (MPO) (Thermo Fisher Scientific, catalog RB-373-A) using the indirect immunoperoxidase method. The MPO-stained neutrophils were counted using $\times 40$ objective in portal tracts and lobular parenchyma in 5 fields with highest density of staining. The mean number of neutrophils per high-power field was calculated. Cells were counted only when nuclei were identified along with cytoplasmic staining, and discarded when only granular staining without identifiable nuclei was seen. Serum aminotransferase levels were obtained at the time of the histologic specimen collection.

Preparation of the liver NPC suspension and FACS. Mouse liver was perfused with HBSS via the portal vein immediately prior to harvesting from animals. Tissues were cut into small pieces and passed through a $40-\mu \mathrm{m}$ nylon cell strainer, and the cells were further centrifuged at $40 \mathrm{~g}$ for 3 minutes to pellet parenchymal cells. The single-cell suspensions were then isolated by Percoll gradient in order to collect the NPC fraction. Cells were incubated with antibodies against CD45-APC (clone 30-F11), mouse Ly-6G (Gr-1) Alexa Fluor 488 (clone RB6-8C5), CD3e-PE (clone 145-2c11), F4/80-Alexa Fluor 488 (clone BM8), and/or mouse CD11b-PE (clone M1/70) (all from BD Biosciences) in FACS buffer for 30 minutes. The cells were analyzed using flow cytometry. The dead cells were excluded by propidium iodide staining.

Statistics. Data are presented as mean $\pm \mathrm{SD}$. Differences between experimental groups were assessed for significance using 1-way ANOVA. Two-tailed Student's $t$ test was used to calculate the $P$ value. A $P$ value less than 0.05 was considered to be statistically significant.

Study approval. All animal studies were reviewed and approved by the board of the Institutional Animal Care and Use Committee of Yale University.

\section{Author contributions}

SYC and JLB conceived and designed the study. SYC, XO, CJS, AM, DJ, and JLB acquired the data. SYC, XO, WZM, and JLB analyzed and interpreted the data. SYC, XO, CJS, WZM, and JLB drafted the manuscript. YC, JW, AM, and YW provided technical/material support.

\section{Acknowledgments}

We thank Fred Gorelick for his helpful editing of the manuscript and Kathy Harry, Jin Chai, Matthew Smith, and Irma Garcia-Martinez for technical assistance. We also thank Themis R. Kyriakides (Yale University) for providing $C \mathrm{cl} 2^{-/-}$breeding pairs, Ruslan Medzhitov (Yale University) for providing

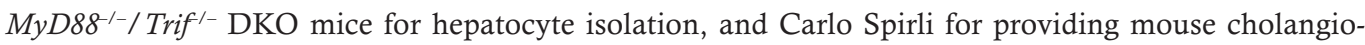
cytes. This work was supported by NIH grants DK25636 (to J.L.B.), DK34989 (to the Yale Liver Center), VA Merit award and NIH grant 2R56DK0 5U01AA021912-02 (to W.Z.M.), and CAMS Initiative for Innovative Medicine grant 2016-I2M-1-011 (to J.W. and Y.W.). 
Address correspondence to: Shi-Ying Cai or James L. Boyer, Liver Center, Yale University School of Medicine, 333 Cedar Street, 1080 LMP, New Haven, Connecticut 06520, USA. Phone: 203.785.3150; E-mail: shi-ying.cai@yale.edu (S.Y. Cai). Phone: 203.785.5279; E-mail: james.boyer@yale.edu (J.L. Boyer).

1. Li T, Chiang JY. Bile acids as metabolic regulators. Curr Opin Gastroenterol. 2015;31(2):159-165.

2. Malhi H, Guicciardi ME, Gores GJ. Hepatocyte death: a clear and present danger. Physiol Rev. 2010;90(3):1165-1194.

3. Faubion WA, et al. Toxic bile salts induce rodent hepatocyte apoptosis via direct activation of Fas. J Clin Invest. 1999;103(1):137-145.

4. Higuchi $\mathrm{H}$, et al. The bile acid glycochenodeoxycholate induces trail-receptor 2/DR5 expression and apoptosis. J Biol Chem. 2001;276(42):38610-38618.

5. Fickert P, et al. 24-norUrsodeoxycholic acid is superior to ursodeoxycholic acid in the treatment of sclerosing cholangitis in Mdr2 (Abcb4) knockout mice. Gastroenterology. 2006;130(2):465-481.

6. Zhang Y, Hong JY, Rockwell CE, Copple BL, Jaeschke H, Klaassen CD. Effect of bile duct ligation on bile acid composition in mouse serum and liver. Liver Int. 2012;32(1):58-69.

7. Woolbright BL, Antoine DJ, Jenkins RE, Bajt ML, Park BK, Jaeschke H. Plasma biomarkers of liver injury and inflammation demonstrate a lack of apoptosis during obstructive cholestasis in mice. Toxicol Appl Pharmacol. 2013;273(3):524-531.

8. Woolbright BL, et al. Bile acid-induced necrosis in primary human hepatocytes and in patients with obstructive cholestasis. Toxicol Appl Pharmacol. 2015;283(3):168-177.

9. Saito JM, Maher JJ. Bile duct ligation in rats induces biliary expression of cytokine-induced neutrophil chemoattractant. Gastroenterology. 2000;118(6):1157-1168.

10. Gujral JS, Farhood A, Bajt ML, Jaeschke H. Neutrophils aggravate acute liver injury during obstructive cholestasis in bile ductligated mice. Hepatology. 2003;38(2):355-363.

11. Allen K, Jaeschke H, Copple BL. Bile acids induce inflammatory genes in hepatocytes: a novel mechanism of inflammation during obstructive cholestasis. Am J Pathol. 2011;178(1):175-186.

12. Sasaki M, Miyakoshi M, Sato Y, Nakanuma Y. Chemokine-chemokine receptor CCL2-CCR2 and CX3CL1-CX3CR1 axis may play a role in the aggravated inflammation in primary biliary cirrhosis. Dig Dis Sci. 2014;59(2):358-364.

13. El-Faramawy AA, El-Shazly LB, Abbass AA, Ismail HA. Serum IL-6 and IL-8 in infants with biliary atresia in comparison to intrahepatic cholestasis. Trop Gastroenterol. 2011;32(1):50-55.

14. Bohan A, Chen WS, Denson LA, Held MA, Boyer JL. Tumor necrosis factor alpha-dependent up-regulation of Lrh-1 and Mrp3(Abcc3) reduces liver injury in obstructive cholestasis. J Biol Chem. 2003;278(38):36688-36698.

15. Seki E, et al. CCR2 promotes hepatic fibrosis in mice. Hepatology. 2009;50(1):185-197.

16. O'Brien KM, Allen KM, Rockwell CE, Towery K, Luyendyk JP, Copple BL. IL-17A synergistically enhances bile acid-induced inflammation during obstructive cholestasis. Am J Pathol. 2013;183(5):1498-1507.

17. Barber GN. Cytoplasmic DNA innate immune pathways. Immunol Rev. 2011;243(1):99-108.

18. Zhang Q, et al. Circulating mitochondrial DAMPs cause inflammatory responses to injury. Nature. 2010;464(7285):104-107.

19. Botla R, Spivey JR, Aguilar H, Bronk SF, Gores GJ. Ursodeoxycholate (UDCA) inhibits the mitochondrial membrane permeability transition induced by glycochenodeoxycholate: a mechanism of UDCA cytoprotection. J Pharmacol Exp Ther. 1995;272(2):930-938.

20. Vaz FM, et al. Sodium taurocholate cotransporting polypeptide (SLC10A1) deficiency: conjugated hypercholanemia without a clear clinical phenotype. Hepatology. 2015;61(1):260-267.

21. Slijepcevic D, et al. Impaired uptake of conjugated bile acids and hepatitis b virus pres1-binding in na(+) -taurocholate cotransporting polypeptide knockout mice. Hepatology. 2015;62(1):207-219.

22. Schoemaker MH, et al. Resistance of rat hepatocytes against bile acid-induced apoptosis in cholestatic liver injury is due to nuclear factor-kappa B activation. J Hepatol. 2003;39(2):153-161.

23. Woolbright BL, Jaeschke H. Therapeutic targets for cholestatic liver injury. Expert Opin Ther Targets. 2016;20(4):463-475.

24. Gäbele E, et al. Role of TLR9 in hepatic stellate cells and experimental liver fibrosis. Biochem Biophys Res Commun. 2008;376(2):271-276.

25. Spivey JR, Bronk SF, Gores GJ. Glycochenodeoxycholate-induced lethal hepatocellular injury in rat hepatocytes. Role of ATP depletion and cytosolic free calcium. J Clin Invest. 1993;92(1):17-24.

26. Denk GU, et al. Tauro- $\beta$-muricholic acid restricts bile acid-induced hepatocellular apoptosis by preserving the mitochondrial membrane potential. Biochem Biophys Res Commun. 2012;424(4):758-764.

27. Carey EJ, Lindor KD. Current pharmacotherapy for cholestatic liver disease. Expert Opin Pharmacother. 2012;13(17):2473-2484.

28. Sombetzki M, et al. 24-nor-ursodeoxycholic acid ameliorates inflammatory response and liver fibrosis in a murine model of hepatic schistosomiasis. J Hepatol. 2015;62(4):871-878.

29. Carchman EH, et al. Experimental sepsis-induced mitochondrial biogenesis is dependent on autophagy, TLR4, and TLR9 sig naling in liver. FASEB J. 2013;27(12):4703-4711.

30. Kim ND, Moon JO, Slitt AL, Copple BL. Early growth response factor-1 is critical for cholestatic liver injury. Toxicol Sci. 2006;90(2):586-595

31. Marra F, Tacke F. Roles for chemokines in liver disease. Gastroenterology. 2014;147(3):577-594.e1.

32. Gehring S, et al. Kupffer cells abrogate cholestatic liver injury in mice. Gastroenterology. 2006;130(3):810-822.

33. Garcia-Martinez I, et al. Hepatocyte mitochondrial DNA drives nonalcoholic steatohepatitis by activation of TLR9. J Clin Invest. 2016;126(3):859-864.

34. Cai SY, Mennone A, Soroka CJ, Boyer JL. Altered expression and function of canalicular transporters during early development of cholestatic liver injury in Abcb4-deficient mice. Am J Physiol Gastrointest Liver Physiol. 2014;306(8):G670-G676.

35. Cai SY, Gautam S, Nguyen T, Soroka CJ, Rahner C, Boyer JL. ATP8B1 deficiency disrupts the bile canalicular membrane 
bilayer structure in hepatocytes, but FXR expression and activity are maintained. Gastroenterology. 2009;136(3):1060-1069.

36. Ouyang X, et al. Adenosine is required for sustained inflammasome activation via the $\mathrm{A}_{2} \mathrm{~A}$ receptor and the HIF-1 $\alpha$ pathway. Nat Commun. 2013;4:2909.

37. Spirli C, et al. ERK1/2-dependent vascular endothelial growth factor signaling sustains cyst growth in polycystin-2 defective mice. Gastroenterology. 2010;138(1):360-371.e7.

38. Torchinsky MB, Garaude J, Martin AP, Blander JM. Innate immune recognition of infected apoptotic cells directs T(H)17 cell differentiation. Nature. 2009;458(7234):78-82.

39. Jennings RT, Knaus UG. Neutrophil migration through extracellular matrix. Methods Mol Biol. 2014;1124:209-218. 